\title{
Analysis of Fluid-Epsilon Oscillation with Simple Harmonic Oscillation
}

\author{
Do Hoon Kim, Shin Hyo Bang, and Taeseon Yoon
}

\begin{abstract}
While studying fluid dynamics, a particular experiment aroused the authors' interest. The experiment is about the attachment of a light sheet of paper to a wide disk of which the center of it is connected to a straw that the experiment performer blows their breath in. This experiment, at a macro level, shows quite a simple result; the paper sticks to the disk against the pressure of exhaled air. At a micro level, however, it shows comparatively complicated result than at the macro level; the sheet of paper inevitably oscillates with a certain period. This phenomenon has not analyzed by any other researchers. On this paper, this phenomenon, which is named as "Fluid-Epsilon Oscillation", is analyzed with simple harmonic motion. Considering of the practical use of the fluid-epsilon oscillation, electromagnetic induction caught authors' mind. Applied to fast moving vehicles, this phenomenon is anticipated to be an efficient solution to the imminent issue of the energy exhaustion. In an attempt to apply the phenomenon to computer science, the authors created a program that calculates the period of the oscillation.
\end{abstract}

Index Terms_Law of continuity, Bernoulli's equation, simple harmonic oscillation.

\section{INTRODUCTION}

In this paper, a particular oscillation of a sheet of paper that is related to the flow of fluid is analyzed by two perspectives. [1] The phenomenon at a macro level is perfectly explained by the law of continuity and Bernoulli's Equation, which will be discussed briefly. The phenomenon at a micro level is unique and has never been studied by other researchers inspired by the fact that the paper has a particular period of oscillation, the authors analyzed the phenomenon with simple harmonic oscillation and considered of its practical application to energy generating.

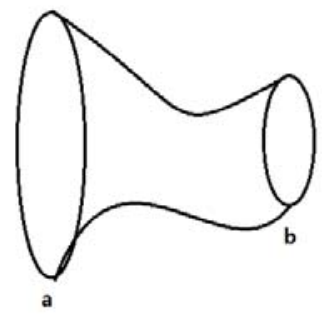

Fig. 1. Law of continuity.

The importance of computer in the field of natural science is increasing; this year, three U.S. scientists won the Nobel chemistry prize on for pioneering work on computer

Manuscript received March 7, 2014; revised May 9, 2014.

The authors are with Hankuk Academy of Foreign Studies, Korea (e-mail: kimdohoon465@naver.com, tsyoon@hafs.hs.kr). programs. In gear with the rapid development of computer technology, the authors decided to predict the oscillation in the virtual reality and built a calculating model based on php.

\section{EXPERIMENT OBJECT}

\section{A. Law of Continuity [2]}

The continuity equation accounts for the flow of ideal fluid. To understand the continuity equation, the comprehension of the peculiarity of ideal fluid should be preceded. Among various characteristics of ideal fluid, the particular quality that is needed to understand the continuity equation is its incompressible nature. Hence, the volume of liquid that flows through a tube does not change.

Assume that the cross sectional area of the object in Fig. 1 at the point $a$ is $A$. That at the point $b$ is $A^{\prime}$. The speed of fluid at the point $a$ is $v$ and at the point $b$ is $v^{\prime}$. In a sufficiently short amount of time, $d t$, the volume of fluid that passes the point is $A v d t$ and $A^{\prime} v^{\prime} d t$ respectively. Since the fluid is incompressible as it was assumed, $A v d t=A^{\prime} v^{\prime} d t$ should be attained. As a result, the continuity equation $A v=A^{\prime} v^{\prime}$ could be derived.

\section{B. Bernoulli's Equation [3], [4]}

According to equation of continuity, the speed of the fluid is variable depending on the vessel of the fluid. When the fluid is in a static state, the pressure depends on the height and velocity of the fluid. Bernoulli's Equation is a significant equation that is regard to the pressure, velocity and the height of ideal fluid. This equation is a fundamental principle of fluid operated pumps, generation of hydroelectric power, and a theory of aviation.

Velocity dependence of pressure can be derived from equation of continuity. When an incompressible fluid pass through a vessel whose cross sectional area varies, the fluid is accelerated, since the velocity of the fluid should vary. When the vessel is horizontal, the force that makes this acceleration should be exerted by surrounding fluid. This means that the pressure should be different depending on the position. If the pressure is all same at every position, the net force exerted on the fluid should be zero. When cross sectional area of the vessel becomes narrow and the speed of the fluid gets higher, to make the net force exerted in the direction of acceleration, the fluid should move from the area where the high pressure exerted to that where the low pressure exerted. Variation of altitude makes additional pressure difference.

$$
P+\rho g h+\frac{1}{2} \rho v^{2}=c o n s \tan t
$$




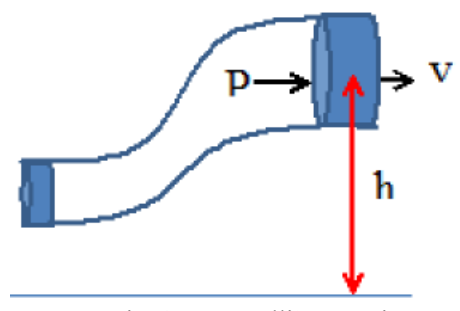

Fig. 2. Bernoulli's equation.

Fig. 2 describes Bernoulli's Equation. $P$ is the pressure of the fluid and $\mathrm{h}$ is the height. $g$ is gravitational acceleration and $\mathrm{v}$ is the velocity of the fluid. $P$ represents the density. This equation means that the sum of the pressure, the potential energy and the kinetic energy of the fluid is constant, when the fluid flows drawing a line of flow.

\section{ANALYSIS OF FLUID-EPSILON OSCILLATION}

\section{A. At a Macro Level}

Figure $\#$ is a simple mimetic diagram of experiment of fluid-epsilon oscillation.

Even the experiment performer blows breath in the straw, the paper sticks to the disk. The principle is quite simple.[1]

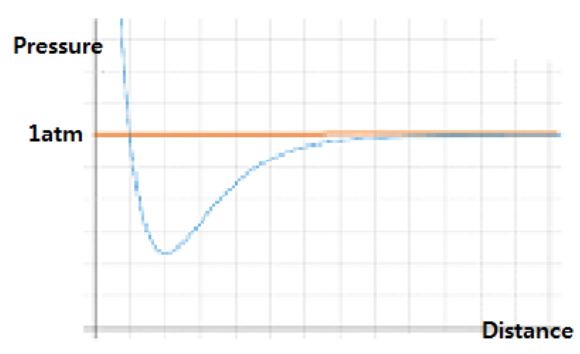

Fig. 3. Distance-pressure graph.

Fig. 3 shows the pressure in accordance with the distance from the axis (straw). Since the circumference of the disk increases as the radius (the distance from the axis) increases, the cross sectional area through which the air particles blow widens. From the equation of continuity, particle's speed slows down as the radius increases. Hence, from the Bernoulli's rule, the pressure increases as the radius increases except for the limited area which is highly affected by the exhaled breath.

At the moment when the air particles are pushed out, they form a jet, which means the pressure of the air blew out from the gap is $1 \mathrm{~atm}$. If not, instead of a jet, there would be a spread of air particles caused by the pressure difference.

As a result, the pressure in the gap between the two faces is under $1 \mathrm{~atm}$. Accordingly, the paper is pushed by the pressure difference between the gap and atmosphere against the exhaled breath.

\section{B. At a Micro Level}

Fig. 4 describes the paper's going down since gravity pulls the paper down. When we blow air into the straw, the air pass through the space between the CD and the paper. Since the velocity of the air increase, the pressure of the space decrease by Bernoulli's equation. Then, the pressure under the paper is atmospheric pressure and the pressure between the $\mathrm{CD}$ and the paper becomes lower than atmospheric pressure. It makes the paper goes up to the CD.

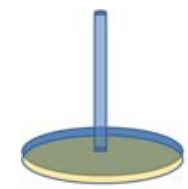

Fig. 4. Fluid-epsilon oscillation.

When the paper goes near the $\mathrm{CD}$, the force that makes the paper go up decrease since the force depends on the distance from the CD to the paper (This is explained by the formula $(*)$ ). Consequently, gravity exceeds the upward force. Then, upward velocity decrease and eventually the paper go down. As the distance from the $\mathrm{CD}$ to the paper increases, the upward force increases and the downward velocity of the paper decreases. Then, the paper goes up again. In this way, the paper oscillates up and down.

A formula that shows the exact period of oscillation and voltage could be derived from regarding the oscillation as simple harmonic motion, since the strength of stability increase when the paper becomes far from the equilibrium point. To calculate the period of oscillation, every force exerted on the paper should be analyzed. Upward force could be calculated by multiplying the pressure and the area where the pressure exerted. The area that the air passes through varies depending on the radius of the $\mathrm{CD}$. Thus, integral calculus is essential to calculate the upward force. The author integrated the force and subtracted gravity exerted on the paper. In this way, the net force can be calculated that is the strength of stability.

When calculating the net force, a fact is derived that the net force depends on the distance from the $\mathrm{CD}$ and the paper. The author applied work-kinetic energy theorem to calculate the maximum distance from the $\mathrm{CD}$ and the paper. Since the paper oscillates, its velocity becomes zero when its displacement from the equilibrium point is maximum. Thus, change of kinetic energy from the top to the bottom is zero and work done by the net force is zero. In other words, the amount of work done by the upward force and that of work done by the downward force are the same. An equation could be set up from this fact. Solving this equation, the maximum distance from the $\mathrm{CD}$ and the paper is derived. The maximum of the net force could be calculated from the maximum distance and the maximum of strength of stability could be calculated from Hooke's law. From this, an equation is set up and elastic modulus is derived. Using the elastic modulus and the mass of the paper, the period of the oscillation could be calculated.

In this formula, constant $c$ and $S$ are included. $c$ is the constant from continuity equation. The author calculated it by applying the last velocity of the air and the area that it passes through. $S$ is the constant from the Bernoulli's equation. Since knowing the last pressure and velocity, the author could calculate it.

Lastly, when substituting mass of the paper, the radius of the straw and $C D$, density of the air, and constant of continuity equation and Bernoulli's equation, the precise period of the oscillation is derived and from this we can get 
voltage of this energy development.

\section{INDUCING FORMULA}

\section{A. Variables}

$t:$ time

$m:$ mass of the paper

$g$ : gravitational acceleration

$P_{0}:$ atmosphere pressure

$P$ : pressure between the paper and the $\mathrm{CD}$

$r_{1}$ : radius of the straw

$r_{2}$ : radius of the $\mathrm{CD}$

$R$ : height of the CD from the surface of the earth

$H$ : maximum distance from the $\mathrm{CD}$ to the paper

$\mathcal{E}:$ minimum distance from the $\mathrm{CD}$ to the paper

$\rho:$ density of the air

$S$ : constant from Bernoulli's Equation

$c:$ constant from continuity equation $(A v=c)$

$v_{0}$ :initial velocity of the air

$P^{\prime}$ : pressure near the straw

\section{B. Calculation of $H$}

When the distance from the CD to the paper is maximum, $\Delta E_{k}=0 \therefore W=0$

$$
\left\{m g+\left(P^{\prime}-P_{0}\right) \pi r_{1}^{2}\right\}\left(h_{\max }-\varepsilon\right)=\int_{\varepsilon}^{h_{\max }} \int_{r_{1}}^{r_{2}} 2 \pi r\left(P_{0}-P\right) d r d h
$$

In (2), the radius of the straw is extremely small compared to that of the CD. Thus, $P^{\prime}$ can be disregarded.

$$
\begin{aligned}
& \left\{\left(m g-P_{0}\right) \pi r_{1}^{2}\right\}\left(h_{\max }-\varepsilon\right)=\int_{\varepsilon}^{h_{\max }} \int_{r_{1}}^{r_{2}} 2 \pi r\left(P_{0}-P\right) d r d h \\
& Y=\left[-\frac{\rho c^{2}}{4 \pi \varepsilon} \ln \frac{r_{2}}{r_{1}}+\pi \varepsilon\left\{\left(P_{0}-S\right)\left(r_{2}^{2}-r_{1}^{2}\right)+P_{0} r_{1}^{2}\right\}-\varepsilon m g\right] \\
& \pm \frac{\sqrt{\left[-\frac{\rho c^{2}}{4 \pi \varepsilon} \ln \frac{r_{2}}{r_{1}}+\pi \varepsilon\left\{\left(P_{0}-S\right)\left(r_{2}^{2}-r_{1}^{2}\right)+P_{0} r_{1}^{2}\right\}-\varepsilon m g\right]^{2}+}}{2 \pi \varepsilon\left\{\left(P_{0}-S\right)\left(r_{2}^{2}-r_{1}^{2}\right)+P_{0} r_{1}^{2}\right\}}
\end{aligned}
$$

In (3), by continuity equation, $c=\pi r_{1}^{2} v_{0}$ Bernouilli's Equation says that $S=P_{0}+\frac{r_{1}^{2} v_{0}}{2 r_{2} h}+\rho g R$

\section{Calculation of Force}

According to the $\pi r_{1}^{2} v_{0}=2 \pi r h v=2 \pi r_{2} h v_{\text {last }}$

$$
v=\frac{r_{1}^{2} v_{0}}{2 r h}
$$

$$
v_{\text {last }}=\frac{r_{1}^{2} v_{0}}{2 r_{2} h}
$$

The potential energy of air molecules is constant. Thus,

$$
\begin{gathered}
\frac{1}{2} \rho v^{2}+P=P_{0}+\frac{r_{1}^{2} v_{0}}{2 r_{2} h} \\
P-P_{0}=\frac{r_{1}^{2} v_{0}}{2 r_{2} h}-\frac{1}{2} \rho \frac{r_{1}^{4} v_{0}^{2}}{4 r^{2} h^{2}} \\
2 \pi r \int_{r_{1}}^{r_{2}}\left(P-P_{0}\right) d r=2 \pi r \int_{r_{1}}^{r_{2}}\left(\frac{r_{1}^{2} v_{0}}{2 r_{2} h}-\frac{\rho r_{1}^{4} v_{0}{ }^{2}}{8 r^{2} h^{2}}\right) d r \\
\therefore F_{\text {total }}=F_{\text {pressure }}+m g=m g+\frac{r_{1}{ }^{2} v_{0}\left(r_{2}-r_{1}\right)}{2 r_{2} h}+\frac{\rho r_{1}^{4} v_{0}{ }^{2}}{8 h^{2}}\left(\frac{1}{r_{2}}-\frac{1}{r_{1}}\right)
\end{gathered}
$$

\section{Simple Harmonic Oscillation Approximation} $F_{\text {total }}=-k x$

$$
m g+\frac{r_{1}^{2} v_{0}\left(r_{2}-r_{1}\right)}{2 r_{2} Y}+\frac{\rho r_{1}^{4} v_{0}^{2}}{8 Y^{2}}\left(\frac{1}{r_{2}}-\frac{1}{r_{1}}\right)=-k \frac{Y-\varepsilon}{2}
$$

The period of simple harmonic oscillation is

$$
\begin{gathered}
T=2 \pi \sqrt{\frac{m}{k}} \\
k=\left(\frac{1}{r_{1}}-\frac{1}{r_{2}}\right) \frac{\rho r_{1}^{4} v_{0}^{2}}{4(Y-\varepsilon) Y^{2}}-\frac{2 m g}{Y-\varepsilon}-\frac{r_{1}^{2} v_{0}\left(r_{2}-r_{1}\right)}{r_{2}(Y-\varepsilon) Y}(6)
\end{gathered}
$$

E. Results

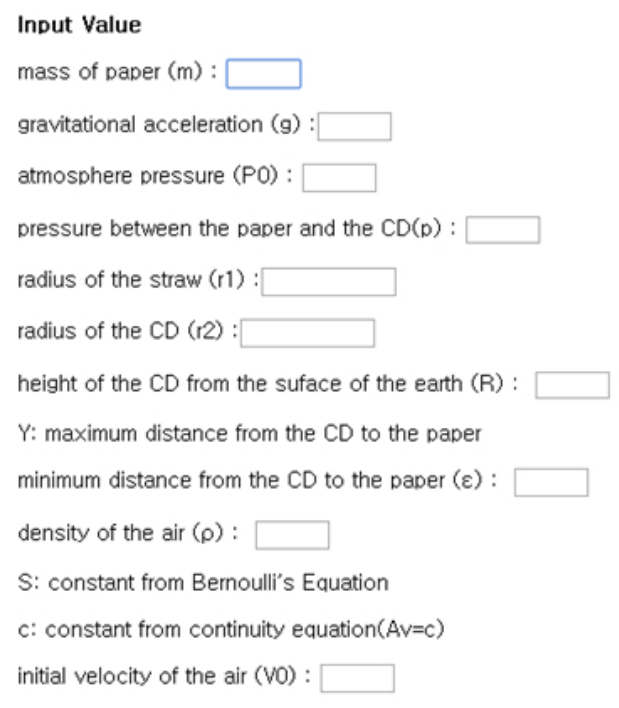

Fig. 5. Oscillation estimation program.

The author programmed the calculator shown as Fig. 5 shown as figure that shows the period of the oscillation when put in certain value of variables [5].

Mass of paper $0.075 \mathrm{kgGravitational} \mathrm{acceleration:}$ $.8 \mathrm{~m} / \mathrm{s}^{2}$

Atmosphere pressure: $101300 \mathrm{~Pa}$

Radius of the straw: $0.0005 \mathrm{~m}$

Radius of the CD: $0.075 \mathrm{~m}$

Height of the CD: from the surface of the earth $1 \mathrm{~m}$ Minimum distance from the $\mathrm{CD}$ to the paper: $0.0001 \mathrm{~m}$ 
Density of the air: $1.275 \mathrm{~kg} / \mathrm{m}^{3}$

Initial velocity of the air: $0.5 \mathrm{~m} / \mathrm{s}$

Pressure between the paper and the CD: $303900 \mathrm{~Pa}$

T: $0.014 \mathrm{sec}$

This period of oscillation cannot be observed by naked eye. Thus, the paper seems to be attached to the CD.

\section{CONCLUSION}

\section{A. Results}

In this paper, a particular oscillation which is named "Fluid-Epsilon Oscillation" is analyzed by two perspectives. The microscopic analysis of this phenomenon is unique and has never been studied until the authors analyzed the phenomenon with simple harmonic oscillation

\section{B. Expectations}

The fluid-epsilon oscillation would be used as a fundamental power source when it is attached to a moving vehicle, which could provide consistent flow of air to the oscillator.

The generator model is comparatively simple than the formula. The straw part is winded around by coil, and magnet is attached to the sheet which plays a role as the paper. The oscillation would let electromagnetic induction to happen.

Our next goals are; first, to build the actual generator model, and second, to analyze the fluid-epsilon oscillation more accurately. Furthermore, a sophisticated experiment that observes the micro level oscillation should be conducted. And the comparison process between the real values and the ones that are calculated by the program should be done.

\section{REFERENCES}

[1] A. Shapiro. (2009). Fluid Mechanics. (Pressure Fields and Acceleration part 2). [Online]. Available: http://youtube.or.kr.

[2] Continuity Equation. (November, 2013). [Online]. Available: www.wikipedia.org.

[3] Young Freedman, College Physics, 10th Ed., vol. 1, Addison-Wesley, 2005, ch. 15 , pp. 250

[4] B. R. Munson, D. F. Young, and T. H. Okiishi, Fundamentals of Fluid Mechanics, $4^{\text {th }}$ ed., John Wiley \& Sons Canada, Ltd., 2002, ch. 3.8, pp. 137.

[5] R. Nixon, Learning PHP, My SQL, JavaScript, and CSS: A Step-by-Step Guide to Creating Dynamic Websites, $2^{\text {nd }}$ ed., O'Reilly Media, 2012, ch. 1, pp. 5.

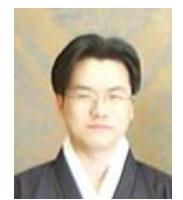

Taeseon Yoon was born in Seoul, Korea, in 1972. He was $\mathrm{Ph} . \mathrm{D}$. candidate degree in computer education from the Korea University, Seoul, Korea, in 2003.

From 1998 to 2003, he was with EJB analyst and SCJP. From 2003 to 2004, he joined the Department of Computer Education, University of Korea, as a Lecturer and Ansan University, as an Adjunct professor. Since December 2004, he has been with the Hankuk Academy of Foreign Studies, where he was a Computer Science and Statistics Teacher. He was the recipient of the Best Teacher Award of the Science Conference, Gyeonggi-do, Korea, 2013.

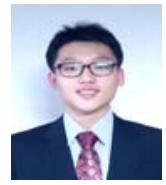

Shin Hyo Bang was born in 1997. He is currently a student in science major of Hankuk Academy of Foreign Studies, Korea. He is mostly interested in energy resources, more specifically the use of Nuclear Energy. He has been studying fluid dynamics and computer programming.

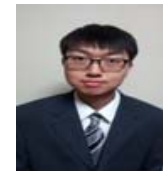

Do Hoon Kim was born in Incheon, Korea, in 1996. He is currently a student in science major of Hankuk Academy of Foreign Studies, Korea. $\mathrm{He}$ is mostly interested in mechanization and aviation. He has been studying fluid dynamics and computer programming. 